\title{
DIÁLOGOS TEMPESTIVOS: LA ADOLESCENCIA Y LAS DETERMINANTES CAPITALISTAS INVOLUCRADAS EN EL CONSUMO DE DROGAS
}

\section{A MANDATORY DIALOGUE: ADOLESCENT AND CAPITALIST VARIABLES INVOLVED IN DRUG CONSUMPTION}

Jaime Alonso Caravaca Morera*

RESUMEN

En los últimos años, se ha atestiguado el aumento significativo en el consumo de sustancias psicoactivas en todo el mundo, principalmente en los países en vías de desarrollo. Este hecho es comprobado por las múltiples investigaciones que revelan la temática, sin embargo, pocos estudios abordan este fenómeno desde la perspectiva del capitalismo y su impacto en los adolescentes. El siguiente artículo formula como objetivo discutir las principales dimensiones del consumo de drogas en la población adolescente, desde la perspectiva del nuevo orden capitalista y la conocida cuestión social.

PALABRAS CLAVE: ADOLESCENTES * TOXICOMANÍA * CAPITALISMO * PROBLEMAS SOCIALES

ABSTRACT

In recent years, we have witnessed a significant increase in the use of psychoactive substances worldwide mainly in sub-developing countries. This fact is proven by the many investigations, however, few studies addressed this phenomena from the perspective of capitalism and its impact on teens. The following manuscript is aimed at discussing the main dimensions of the drug use among adolescents from the perspective of the new capitalist order and the known social issue.

KEYWORDS: ADOLESCENTS * DRUG ABUSE * CAPITALISM * SOCIAL PROBLEMS

Universidade Federal de Santa Catarina, Florianópolis, Brasil.

jacamorera@hotmail.com. 


\section{INTRODUCCIÓN}

El consumo de drogas ha acompañado la historia de la humanidad durante su proceso evolutivo; ya que el ser humano siempre mantuvo una relación bastante estrecha con sustancias psicoactivas por diversos motivos, algunos de ellos culturales, religiosos, recreativos, de enfrentamiento a los problemas, de transgresión, trascendencia, como medio de socialización o aislamiento.

En el campo individual, los diversos efectos provocados por el uso/abuso de drogas (tanto lícitas como ilícitas), resultan incontestables; se pueden asumir condiciones altamente perjudiciales, desde una perspectiva biológica, psicológica y social. Las proporciones de tales consecuencias constituyen en la actualidad niveles alarmantes $y$ justifican los innumerables esfuerzos emprendidos en función de reducir los daños causados por ese consumo.

Desde el punto de vista teórico, se destaca en líneas generales dos corrientes epistemológicas que procuran ocuparse de las discusiones sobre la temática en cuestión: se denomina a la primera como corriente moderna (se incluye lo que algunos llaman perspectiva posmoderna) que tiene su foco de atención dirigido, predominantemente, al espacio político y criminalístico del fenómeno. Aquí se insieren las diversas investigaciones que evidencian datos epidemiológicos, políticas, normativas, acciones y estrategias de punición (Dávila, 2005 y da Silva, 2013).

Aunque se reconozca la importancia de tales contribuciones para el conocimiento científico, se considera que el enfoque de las propuestas presentadas por los estudios de esta vertiente teórica se encaminan hacia el mejoramiento de los números divulgados por los entes estatales y para responsabilizar a los individuos, mediante una comprensión superficial en que no es posible construir otro proyecto de sociedad y que, por tanto, como máximo puede alcanzarse una mejoría de las cifras endémicas $y$ de incidencia/prevalencia del fenómeno, a través de acciones que posibiliten únicamente la reducción de oferta y demanda en la contemporaneidad.
La segunda vertiente teórica, que se denomina de perspectiva crítica, procura revelar los diversos aspectos presentes en la cuestión del uso/abuso de alcohol, tabaco y otras drogas con base en el estudio del modelo social vigente, en cuya raíz se sitúa la explotación del hombre por el hombre, cuyos reflejos exceden la esfera numérico-económica, y se expresan en todas las demás dimensiones sociales. Según esta perspectiva, la cuestión en debate se expone en cuanto fenómeno social, plasmado en un complejo societario, cuyo análisis crítico evidencia la necesidad de su superación al reconocer que, aun cuando momentáneamente las acciones más inmediatas puedan traer alguna contribución, estas no consideran la comprensión de la totalidad ontológica y gnoseológica de las drogas y sus usuarios, en este caso de los adolescentes (Dávila, 2005 y da Silva, 2013).

Por otra parte, la adolescencia representa un proceso tanto de deconstrucción como de reconstrucción y afirmación de la identidad. Dicho proceso puede ser influenciado por conflictos interiores generados a lo largo de su decurso, una vez que el adolescente pasa por momentos de nuevas experiencias, reformulación de (auto) conceptos, síntesis de las victorias y pérdidas de la infancia, reformulaciones de carácter social, cuestiones de sexualidad, selecciones relacionadas con la vocación profesional, entre otros aspectos característicos de esta fase.

Este proceso puede ser considerado como un continuum en el desarrollo humano, que contempla una fase de transición entre la infancia y la vida adulta, constituyéndose en un mecanismo multidinámico, en el cual se desencadena una serie de transformaciones profundas e intensas en las esferas físicas, psicológicas $y$ sociales que muchas veces puede promover espacios laberínticos de experimentación de sustancias psicoactivas y uso/abuso de drogas.

De esta manera, al (re)conocer a la adolescencia como una de las etapas críticas de entrada y experimentación del uso/abuso de drogas en el mundo contemporáneo, el presente texto pretende efectuar una reflexión sobre las dimensiones participantes en el consumo de drogas por parte de los adolescentes. Esta 
discusión fue construida con el soporte teórico de los principales investigadores brasileños y latinoamericanos de trayectoria en las áreas de Enfermería, Antropología, Sociología y Salud Colectiva, al tiempo que presenta algunas consideraciones teórico-conceptuales que visan contribuir con los debates del sector socio-sanitario.

La presente reflexión articula de manera lógica, miradas, elementos y conceptos defendidos por diferentes autores, para retratar el estado del arte relacionado con la temática de consumo de drogas lícitas e ilícitas por parte de los adolescentes y sintetizar racionalmente las referencias seleccionadas, con el fin de continuar el debate dentro $y$ fuera de nuestras fronteras físicas y simbólicas. De este modo, se destaca que el proceso de búsqueda de datos y selección de los investigadores fue intencional y no exhaustivo, asimismo, el análisis reflexivo nace producto de la lectura crítica de los textos, conducido por el autor principal.

\section{EL USO/ABUSO DE DROGAS COMO FENÓMENO SOCIAL}

A partir de los cambios, desafíos y retos que la adolescencia ofrece con sus artimañas de bombardeo hormonal y contextual, el joven parece estar más vulnerable para experimentar $y$ crear dependencia de alguna sustancia psicoactiva (sea esta legal o ilegal).

Como se ha mencionado, se considera que el uso/abuso de drogas acompaña el proceso evolutivo de todas las civilizaciones, aunque la relación establecida varíe entre las sociedades y los períodos históricos. Sin embargo, se resalta que su consumo pandémico constituye una característica de tiempos más recientes y al tomar en cuenta que el deseo de trascender es inherente al ser humano, se verifica que ese deseo consiste en un riesgo y un peligro más identificable durante el período de la adolescencia.

Analizar las diferentes formas de uso/ abuso de drogas, así como, los factores que estarían asociados en la población adolescente, impone explícitamente comprender las múltiples dimensiones $y$ determinantes socioculturales y político-económicas que definen dicho fenómeno social.
El fenómeno de las drogas se encuentra constantemente en la pauta de las discusiones políticas y entre académicos de las más diversas áreas. Esto como consecuencia de sus devastadores efectos societarios, así como, su indistinción en relación con las clases sociales y principalmente por tratarse de una más, entre las contradicciones del sistema neocapitalista.

Por un lado, este fenómeno es el responsable de movilizar un intenso y lucrativo mercado (que demuestra el incontrolable dominio del capital sobre las relaciones sociales, como reflejo de la mercantilización del ser, estar y poseer en la vida de los individuos) y por otra parte, representa a través de su aspecto político y social, una contra-amenaza al propio orden capitalista (da Silva, 2013).

Aunque el uso/abuso de drogas por parte de adolescentes no resulte exclusivo de una determinada clase social, las razones y circunstancias parecen peculiares para cada grupo social. Esto significa que en las regiones más pauperizadas, el uso de drogas por adolescentes puede relacionarse con las condiciones económicas precarias y la falta de oportunidades para mejores condiciones de vida. Por otro lado, entre los adolescentes que poseen una condición económica más ventajosa, el exceso de libertad y la falta de supervisión/diálogo familiar, parecen constituirse en factores importantes para el uso de drogas, según un estudio realizado en el año 2011, con 900 adolescentes, en una ciudad de la región nordeste de Brasil (MedinaArias y Ferriani, 2010; da Silva, 2011).

Dicho estudio resaltó también que dentro de los factores que influenciaban la entrada en el mundo laberíntico de las drogas, estuvieron asociados: la violencia familiar (específicamente la física, psicológica, sexual y abandono/negligencia), los divorcios, la influencia de los grupos de amigos, el tipo de entretenimiento practicado en los momentos de ocio, la muerte de una persona significativa $y$ la falta de espiritualidad.

Otro estudio desarrollado en México por Velázquez, Arrellanes y Martínez (2012) resalta que el consumo de sustancias psicoactivas no acontece por falta de información, sino que se deben explorar todas las variables apuntadas en 
la investigación brasileña y que se encuentran circunscritas en su definición; debido a que la mayoría de los adolescentes tienen un conocimiento básico sobre el uso de drogas y sus consecuencias. Por esta razón, es necesario llevar a cabo una mirada más crítica que permita visualizar la génesis del fenómeno del uso/abuso del alcohol, tabaco y otras drogas en la población adolescente.

Este fenómeno no puede comprenderse apenas en su carácter subjetivo, sino que precisan ser consideradas las determinaciones concretas de la realidad inmersas en la relación dialéctica con los sujetos y esta no es una peculiaridad que ocurra únicamente con los adolescentes, mas sí una condición ontológica de los seres humanos, indispensable para el análisis de los diversos fenómenos sociales.

El uso/abuso de drogas representa otra de las máculas sociales engendradas en el modo de producción capitalista. Por eso, se pretende discutir sobre su determinación material y social, además de debatir acerca de las tendencias teóricas y políticas que procuran interpretar tal fenómeno. Alcanzar este audaz objetivo exige una interpretación crítica de la realidad y esto solamente es posible a partir de una perspectiva que considere la constitución histórico-dialéctica del fenómeno.

La literatura que trata sobre el uso de drogas, permite percibir que existe una relación estrecha del fenómeno en cuestión (en la vida del adolescente) con diversos males sociales, como violencia, desempleo, educación deficiente, pobreza, entre otros. Además, esas expresiones de deterioro social poseen una génesis en común, ya que comparecen en el complejo totalitario como expresiones de la "cuestión social", considerando que esta es única y exclusivamente originada en la sociedad capitalista (Marques y Cruz, 2000; Schenker y Minayo, 2005; da Silva, 2011 y Marx, 2005).

Se utiliza en este texto el término de la "cuestión social" entre comillas por comprender que tal expresión no resulta semánticamente unívoca, al contrario, se registran en torno a esta, comprensiones diferenciadas $y$ atribuciones de sentido muy diversas. Esa expresión tiene una historia reciente, su uso parece haber iniciado en la tercera década del siglo xix, con el objetivo de contemplar el fenómeno más evidente de la historia de la Europa Occidental, sobre los impactos de la primera ola industrializadora iniciada en el siglo xvin: el fenómeno del pauperismo, que para los más lúcidos observadores de la época, independiente de sus perspectivas teóricas, se tornó claro que consistía en una nueva pobreza.

Por primera vez en la historia, la pobreza crecía en la medida en que aumentaba la capacidad de producir riqueza o sea, cuanto más la sociedad se mostraba capaz de producir bienes y servicios, más aumentaba el contingente de sus miembros que aparte de no tener acceso a tales bienes y servicios, se veían desposeídos de las condiciones materiales de la vida con que contaban anteriormente. En resumen, la pobreza del primer tercio del siglo xix se mostró efectivamente como un proceso renovado, ya que, contradictoriamente, fue producida en las mismas condiciones que propiciaban los supuestos de su supresión. A ese pauperismo y sus desdoblamientos se les designó como "cuestión social" (Marx, 2002).

En ese sentido, los diversos aspectos económico-sociales expresados como resultado de investigaciones que trabajan sobre el consumo de drogas en adolescentes, precisan ser comprendidos a la luz de una teoría que explique, desde el punto de vista histórico y económico, cómo surgen las conformaciones sociales vigentes y cuál es la génesis que no solamente se limita a casos de México, Costa Rica y Brasil, mas remonta la compleja totalidad social y las refracciones de la llamada "cuestión social de América Latina".

Inicialmente, se observa que en el capitalismo, la masa de medios de producción crece con la productividad del trabajo. Esa situación se refleja significativamente en la composición del capital, ya que con la creciente productividad del trabajo, no solamente se eleva el volumen de los medios de producción, también cae el valor de ellos en comparación con su volumen; es decir, su valor se eleva de modo absoluto, pero no proporcionalmente a su magnitud (Marx, 2002). 
Así, acumular capital implica el aumento del proletariado (capaz de generar valor adicional) al paso que el trabajo muerto (de las máquinas y equipos) apenas transfiere valor para el producto final; Marx (2002) afirma que producir más valía constituye la ley absoluta del capital, de este modo de producción. A su vez, la fuerza de trabajo solo es vendible cuando conserva los medios de producción como capital y proporciona, como trabajo no pago, una fuente de capital adicional.

De esta forma, está puesto el sistema de causalidades del pauperismo y consecuentemente, la raíz material de la "cuestión social", gestada en medio de la explotación del trabajo que garantiza la expansión, la acumulación y la reproducción del capital. Netto (2001) corrobora que no existe una nueva "cuestión social" y que se debe investigar más allá de la permanencia de manifestaciones tradicionales de dicho fenómeno (fuerte desigualdad, desempleo, hambre, enfermedades, penuria, etc.), al tomar en cuenta la emergencia de nuevas expresiones de esa cuestión (in)suprimible sin la supresión del orden del capital.

En este sentido, la "cuestión social" asume diferentes rostros en el capitalismo contemporáneo y así todos los males sociales que poseen su base material en la ley general de la acumulación primitiva se consideran expresiones de la "cuestión social". Frente a esta situación, se puede inferir que el consumo de drogas por los adolescentes, de cierto modo puede ser presentado como una expresión de la "cuestión social"; sin embargo, se debe tomar en cuenta que no es un fenómeno exclusivo de la población largada en el pauperismo y consiste en un fenómeno históricamente anterior al sistema capitalista, aunque sus relaciones con la pobreza, el desempleo y la violencia sean claramente perceptibles (Cáceres, Salazar, Varela y Tovar, 2006).

No se puede ignorar que el uso de drogas, anterior al capitalismo, asume particularidades expresivas dentro del mundo capitalista y que los motivos que llevan a una persona con mayor poder adquisitivo podrían resultar diferentes de aquellos que poseen esa característica restricta. No obstante, existe un hilo conductor en común, el uso de drogas sufrió profundas modificaciones con el surgimiento del capitalismo, ya que se creó un comercio lucrativo que solo puede existir dentro de las relaciones sociales de la sociedad burguesa y precisamente dentro de esa estructura societaria (en la cual el mercado está inmerso) se permite a los pobres $y$ a los ricos consumir la mercadería denominada droga.

En ese sentido, lo más correcto es afirmar que el fenómeno social del uso/abuso de drogas está plasmado en las expresiones más básicas de la "cuestión social", ya que consiste claramente en un desdoblamiento del pauperismo y figura como un fenómeno relacionado a este, si se considera que permea todas las clases sociales y posee relación directa con el modo de vivir de la sociedad que produce la necesidad del consumo de drogas con la proporción y la motivación que se percibe hoy.

Como se consignó, este no es un fenómeno exclusivo de la sociedad capitalista, por el contrario, se ha establecido a lo largo de la historia de la humanidad. Jansen (2007) afirma que en ciertas sociedades había un uso social de las drogas, generalmente moderado y vinculado a las prácticas culturales y religiosas. Sin embargo, cuando la droga se convierte en mercadería, se encuentra una dinámica completamente diferente en su utilización: primero, su consumo pasa a colocarse más allá de cualquier marco cultural-religioso; segundo, se torna en la fuente de grandes lucros (de hecho, la producción masiva ocurriría durante la Revolución Industrial, con la transformación del opio en heroína y de la hoja de coca en cocaína al final de siglo xix e inicios del siglo xx).

Se puede mencionar el caso también de la Guerra del Opio, que evidencia la vinculación de la droga con la expansión internacional capitalista y colonial militar. Los portugueses, en el siglo xvi, iniciaron el comercio del opio, sustancia que compraban en la India y vendían principalmente a China. En el siglo xviII, los portugueses fueron sustituidos por los ingleses, quienes lucraron significativamente con el tráfico de opio que desde 1989, casi monopolizaron las finanzas inglesas (Escohotado, 1998). 
De esa forma, el opio enriquecía a Inglaterra y de cierto modo, también movilizaba la economía china, al paso que simultáneamente destruía a su pueblo, lo que presionó al Gobierno chino a no legalizar su comercialización. Los chinos apelaron ante la reina Victoria en relación con los efectos nocivos del tráfico de opio y del robo de oro $y$ plata en su territorio; sin embargo, ella no se mostró favorable a las reivindicaciones solicitadas. Como consecuencia, los chinos comenzaron a destruir los cargamentos de opio, esto llevó a Inglaterra a declarar la guerra: el resultado fue la derrota de China, que se vio forzada a ceder Hong Kong al Estado inglés (Escohotado, 1998).

Por otra parte, se percibe que el modo de producción capitalista impulsa y modifica el uso de drogas, reconfigurándolo y transformándolo en una mercancía destinada explícitamente al mercado consumidor. Por eso se constata que aun cuando la droga estaba presente en las sociedades pre-capitalistas, es en las condiciones objetivas del capitalismo que esta se torna un fenómeno social, convirtiéndose en la causa de la ruina de muchos y la riqueza de otros.

Como reflejo de esto, se encuentra el tráfico de drogas, un gran negocio capitalista organizado para obtener el máximo de lucro, al tiempo que se constituye como un importante mecanismo de destrucción humana. En la base del fenómeno, se halla la explosión del consumo y la popularización de la droga, especialmente en los países más desarrollados. Los más afectados de la sociedad son aquellos que no poseen perspectivas, principalmente los jóvenes inmersos en el desempleo crónico y los hijos de las clases estigmatizadas que sufren de los efectos de la descomposición social y moral (Silva, Micheli, Camargo, Buscatti, Alencar y Formigoni, 2006).

Por lo tanto, se resalta cómo la lógica del comercio de la droga resulta contradictoria (así como el propio capital). Si por un lado se tiene la autodegradación humana y social, por el otro, se observa que el universo del narcotráfico consiste en uno de los negocios más lucrativos en la contemporaneidad.
ARTICULACIÓN DE ADOLESCENCIA Y USO/ABUSO DE DROGAS

Como se ha discutido en este articulo, el uso/abuso de drogas es un fenómeno intrínsecamente inmerso en la "cuestión social" y asume diferentes facetas (con sus diferentes peculiaridades), mediante las clases antagónicas constitutivas de la sociedad burguesa. Aunque el uso de drogas sea históricamente anterior al sistema del capital, es en la sociedad dominada por este, que la droga pasa a prevalecer como mercancía y como tal proporciona una reconfiguración a su uso, que adquiere una nueva dinámica, tornándose en pieza fundamental para el proceso de producción y reproducción del orden socio-metabólico capitalista.

Dentro de este proceso, los adolescentes representan un grupo poblacional fuertemente imbricado en la "cuestión social", al ser comúnmente participantes en la producción, comercialización y consumo. Además, se presentan como público meta de las principales medidas de reparación de las consecuencias de esta problemática.

En ese sentido, se presume que la intrínseca relación entre los adolescentes y el consumo de drogas se insiere en el ámbito de las estrategias utilizadas por el sistema del capital en el atendimiento de sus necesidades permanentes de acumulación y expansión. Es decir, tal relación está irrefutablemente determinada por la totalidad concreta, si se considera que el ser no se trata de una categoría abstracta, en la medida en que se comprende como totalidad concreta, dialécticamente articulada en totalidades parciales o sea, que se presenta por medio de la interacción de los elementos en el interior de cada complejo.

De ahí, se puede afirmar que la relación adolescentes-drogas se presenta como un mecanismo singular mediante la totalidad concreta, en un proceso en que inciden rebatimientos mutuos entre los diferentes complejos (Almeida, Ferreira, Barbosa, da Silva y Santos, 2007).

En esta categoría, se discute sobre algunas totalidades parciales, productoras y mantenedoras de vulnerabilidad de los adolescentes al uso/abuso de drogas. Se pretende, consecuentemente, que una vez comprendidas a partir de 
su raíz material e histórica, las categorías aquí analizadas puedan contribuir para la reconstrucción de una perspectiva que se dirija a crear soluciones reales y efectivas para la problemática en cuestión.

El uso de drogas por parte de adolescentes se coloca como una necesidad social, o sea, antinatural y consecuentemente, condicionada por las relaciones sociales engendradas mediante condiciones objetivas de la sociedad capitalista. En la actualidad, las razones que motivan el uso de drogas en la adolescencia se inscriben de manera predominante dentro del contexto social en el cual el adolescente está inserto.

Contemporáneamente, son diversas las relaciones que sustentan el uso/abuso de drogas como fenómeno social: desde las imposiciones de cuño ideológico hasta sus refracciones para las condiciones materiales de vida, con lo que permean las más variadas instituciones de convivio de los adolescentes, en que destacan la familia y la escuela (Marques y Cruz, 2000).

En este contexto, el primer factor que se señala es la concepción introyectada ideológicamente sobre la adolescencia, desde el sentido común al campo científico. Se sabe que la adolescencia consiste en un período de intensas transformaciones biopsicosociales; sin embargo, el ideal difundido presupone que este momento singular del ciclo vital necesita representar un momento de crisis, desorden e irresponsabilidad. De hecho, el adolescente es visto $y$ tratado como un individuo que vivencia una etapa extremamente conturbada de su madurez y por esa razón sus relaciones familiares, escolares y con todos aquellos de su grupo de convivencia social siempre estarían llenas de conflictos e impasses. Cabe resaltar que los medios de comunicación son uno de los principales responsables por esta manipulación ideológica, acuñada a veces en expresiones peyorativas o poco comprensivas (Riofrio y Nascimento, 2010).

Sin embargo, es preciso observar que en ese contexto de "crisis, desorden e irresponsabilidad" o en los dilemas generados por este, la droga es uno de los elementos más presentes, lo que representa el auge de ese proceso socialmente construido. En ese sentido, el adolescente vivencia un proceso natural de transformación que a pesar de traer consigo una nueva realidad inmersa de dudas y miedos, forma parte de la evolución humana y debe ser vivido con esas características.

Además, se resalta que en la sociedad contemporánea, algunos elementos de las esferas sociopolíticas se encuentran fragilizadas, así como, la familia y la escuela evidencian un proceso de precarización en sus vínculos $y$ funciones sociales, al tiempo que el mercado de drogas se fortalece, tornándose cada vez más lucrativo y que los medios de comunicación/redes sociales y otros mecanismos de socialización crean representaciones sociales de las drogas con múltiples sentidos y significados (Marques y Cruz, 2000; Murillo-Castro y Miasso, 2011).

El contexto expuesto, refleja la condición de recíproca determinación entre los complejos de la totalidad concreta (fragilidad sociopolítica, mercado lucrativo de drogas, precarización de la familia y de la escuela, concepción de desorden de la adolescencia, infraestructura social deficiente $y$ finalmente, la representación social de las drogas), con énfasis para la predominancia de la determinación de la esfera económica, con mayores implicaciones en el actual momento histórico, donde se sitúan las bases materiales del fenómeno del uso/abuso de drogas.

Se puede afirmar que las transformaciones recurrentes de la relación entre el adolescente y el uso de drogas representan, dentro de un análisis de los componentes del macrocomplejo, momentos que se presentan permanentemente en un estado de determinación reflexiva.

Es interesante observar que la ideología del desorden reforzada por el apelo de los medios comunicativos, no incide apenas sobre los adolescentes, esto porque todas las instituciones sociales también forman parte de una estructura responsable de sustentar y difundir las ideologías necesarias para la reproducción del sistema capitalista. En este contexto, se sitúan las relaciones del eterno conflicto entre adolescentes, padres y escuela, que a su vez acaban sin contribuir a una adolescencia emancipatoria $y$ de descubrimientos. Por lo contrario, 
tales relaciones tienden más a fortalecer la concepción de crisis (da Silva, 2011 y 2013; Schenker y Minayo, 2005).

Es importante resaltar también la funcionalidad de la alienación producida a través de la ideología burguesa en el proceso del oscurecimiento de la esencia del fenómeno del uso/ abuso de drogas y de sus consecuencias para el adolescente. En ese campo, se fomentan intervenciones inmediatas sobre el problema, esto permite la manutención de la sociedad desigual (antiisonómica) e injusta, al silenciar sobre las raíces materiales de la "cuestión social", conforme a lo citado anteriormente, cuando se refirió al aspecto predominante en la propuesta de la actuación estatal. De este modo, se compone una cápsula de apariencias que impide al adolescente comprender las particularidades de este proceso dentro de una perspectiva de la totalidad social.

Así pues, la propia adolescencia, como es comprendida en esta sociedad, se configura como un campo fértil $y$ vulnerable para el consumo de drogas, visto por muchos como un libertador de los conflictos familiares, como respuesta para las interrogaciones existenciales o aún como la tan soñada emancipación que esta sociedad no puede proveer. En este contexto, están puestas en líneas generales algunas condiciones fundamentales para el uso cada vez más prematuro de drogas.

Por ejemplo, en el Centro Brasileño de Informaciones sobre Drogas Psicotrópicas se afirma que, en aproximadamente la mitad de los casos, el uso de sustancias psicoactivas se inicia entre los 10 y 12 años. Las constataciones y tendencias apuntadas por diversas investigaciones, ayudan a comprender cómo las vulnerabilidades del adolescente (recurrentes del proceso de transformación biopsicosocial natural de esta fase, más condicionadas por las fragilidades de una sociedad firmada sobre la explotación del ser humano por el ser humano), se expresan en la actualidad, de manera más severa, atingiendo al adolescente en una edad cada vez menor (da Silva, 2011 y 2013; Marques y Cruz, 2000).

Schenker y Minayo (2005) analizan que cuanto más temprano se dé la experimentación de una sustancia psicoactiva por parte de un adolescente, mayores serán las probabilidades de crear una dependencia y como consecuencia, aumentarían las posibilidades de causar mayores repercusiones de vulnerabilidad social y estigmatización. Además, los mismos autores destacan que esa dependencia resulta incentivada principalmente por las modificaciones significativas que las drogas han sufrido a lo largo de la historia. Por ejemplo, la marihuana, en los años de 1970, contenía menos de 0,2\% de тсн (Delta 9-tetrahidrocanabinol) y 20 años después multiplicó considerablemente su contenido, llegando en promedio a poseer un $6 \%, y$ alcanza en la actualidad cantidades aproximadas del $15 \%$ de la sustancia.

Por otra parte, el nuevo ordenamiento social vigente se ocupa de manipular el ideario sobre el uso de drogas, en una tentativa de atender las demandas impuestas por un mercado irrefrenable $y$ evidentemente, una vez instaurada la "ideología de desorden" o momento de "crisis" e "irresponsabilidad", se exponen las condiciones materiales que propician su consumo en la adolescencia.

Al delimitar este espacio en el cual los adolescentes supuestamente se sitúan, algunos elementos sociales (tales como las relaciones familiares negativas, la influencia del grupo de pares, las redes sociales, los medios de comunicación o el deseo de experimentación) pasan a promover algunas estrategias de vulnerabilidad, para alcanzar también a aquellos adolescentes que aún no recurrieron al uso de drogas en este periodo específico o que por cierto factor no lo vivenciaron tan intensamente (Silva et ál., 2006).

Las estrategias utilizadas por los elementos sociales anteriormente mencionados resultan bastante refinadas $y$ en algunas ocasiones se confunden con el principal mecanismo del que se vale la burguesía para tratar el asunto de las drogas, promovidas ampliamente como el "lubricante social". Para este fin, esos elementos usan los más diversos artificios de seducción, como estrategias de poder, diversión, sexualidad, aceptación social y liberación cultural. De este modo, el uso de drogas pasa a representar una condición esencial para el adolescente en casi todos los momentos que vivencia, ya que la recreación, la aceptación social 
y la sexualidad ocuparían posiciones centrales en las relaciones sociales de esta fase de la vida, $y$ son en todo tiempo buscadas $y$ aspiradas.

La influencia de los medios de comunicación y de algunos aparatos sociales (aunque con algunas especificidades) legitiman la práctica del uso de drogas y pasan a construir ideales en el cotidiano del adolescente que no permiten concebir la playa sin cerveza, la fiesta sin bebida destilada, los clubes sin drogas psicodélicas y así sucesivamente, en una constante y evidente asociación de beneficios sociales y naturales sin precedentes (da Silva, 2011).

Un segundo factor que precisa ser considerado en esta relación del adolescente con el uso de drogas, son las condiciones de vida, articuladas desde el proceso de urbanización desenfrenada del país hasta la negación del acceso a las políticas públicas. En ese sentido, en la cuestión social, deben tomarse en cuenta el carácter constitutivo del modo de producción capitalista $y$ las contradicciones que forman parte de este sistema, dirigidas a la generación del lucro y manutención de la clase detentora de los medios de producción (Almeida et ál., 2007).

Es indispensable recordar que en el caso de algunos adolescentes, el desempleo, la desestructura familiar, la educación precaria, la falta del acceso al ocio, a la cultura y al deporte, constituyen factores que inclinan al adolescente hacia el uso de drogas, en la mayoría de los casos por falta de otro modo de participación social o para soportar las adversidades impuestas por el complejo societario excluyente.

Conviene también mencionar que, aun cuando la clase trabajadora es la que más sufre con la cuestión social y sus refracciones, dada la constitución ontológica del ser social, este estado de precarización se expande a todas las relaciones humanas, tanto en un proceso directo de incorporación por el capital como en el caso de la espiritualidad, de la familia y de la escuela. El resultado de esto es que los adolescentes (aunque pertenezcan a una clase económicamente privilegiada) también acaban por vivenciar la realidad de sometimiento de esta sociedad, ya que todo está sumergido en un proceso de precarización y de este modo su condición de clase dominante no consigue protegerlos integralmente de la exposición y del uso de drogas.

Al considerar los antagonistas estructurales e insuprimibles de las dos clases principales de la sociedad capitalista, se observa que también, en lo relacionado con el uso de drogas, los patrones que se presentan son diferentes entre los adolescentes oriundos de la clase dominante y los de la clase trabajadora. Tal división ayuda a reforzar la presentación del uso de droga como un "panacea" y para esto basta recordar que algunas drogas traen consigo representaciones sociales que caracterizan a determinado grupo social (Cáceres et ál., 2006).

Otra arista expuesta en la lectura de la relación entre el adolescente y el uso/abuso de drogas, se configura en el plano comercial, el precio bajo, la libre comercialización de algunos tipos de drogas y la ineficiencia estatal en cohibir otros, además de la exposición en diversos locales que facilitan la publicidad $y$ tornan algunas drogas más accesibles, promoviendo la diseminación del uso indiscriminado de esas sustancias, exponiendo fragilidades del aparato estatal y social en responder las demandas impuestas por la problemática en cuestión en toda su complejidad y magnitud.

\section{CONSIDERACIONES FINALES}

La realidad expuesta en este documento es apenas la punta del iceberg en lo que hoy constituye el comercio de drogas, comprendido desde los latifundios nacionales hasta en los narcotraficantes de espacios internacionales, ambos abastecidos con el lucro proporcionado por la venta de drogas que ocurre bajo la influencia de los medios de comunicación, del Estado y de las vulnerabilidades indicadas anteriormente.

La adolescencia se constituye en un periodo de madurez física y psicológica que implica varias e importantes transformaciones, analizar este fenómeno multidimensional sobre la perspectiva de la cuestión social significa comprender cómo los abordajes interdisciplinares e intersectoriales se configuran como posibilidades de enfoque de esta problemática, por tal razón, delinear e implementar estrategias de participación 
efectiva del adolescente, su familia y todas sus dimensiones en un saber colectivo sobre el fenómeno de drogas resulta fundamental.

Sin lugar a dudas, las fuerzas que impelen los adolescentes al uso/abuso de drogas son más articuladas y más numerosas de las que se oponen. La comprensión de cómo se engendra este enmarañado de totalidades parciales, constituye un punto estratégico para el entendimiento de la totalidad concreta de la vida humana, en particular en lo que respecta al adolescente, al paso que crea sobreposiciones, tornando al adolescente en víctima que precisa luchar diariamente en la tentativa de no caer en las manos de esta práctica laberíntica y engañosa.

Todas las cuestiones aquí levantadas consisten en un intento de desmembrar la determinación del uso/abuso del tabaco, alcohol y otras drogas a la luz del modo de producción capitalista (su raíz material, considerando la singularidad del uso de drogas por el adolescente). Por esta razón, se procuró contribuir con la comprensión de los elementos indispensables en la construcción de una propuesta de sociedad en la cual se revele una adolescencia emancipadora.

La identificación del adolescente en condición de vulnerabilidad en relación con el uso/ abuso de alcohol, tabaco y otras drogas en un entorno capitalista, aún en nuestra contemporaneidad, es un asunto bastante complejo. El reconocimiento de algunas características de riesgo podría auxiliar para la realización de nuevos trabajos que ayuden a la elaboración de nuevas políticas y la ejecución de nuevas normativas que consideren todas las variables macroeconómicas, familiares, escolares, espirituales y socioculturales que se encuentran circunscritas en el fenómeno de las drogas.

\section{REFERENCIAS}

\section{LIBROS}

Da Silva, Sóstenes Ericson Vicente. A questão do uso de drogas por adolescentes. Maceió, Alagoas, Brasil: Edufal, 2011.

Da Silva, Sóstenes Ericson Vicente. Redução de danos e prevenção do uso de drogas: contribuições para práticas socioeducativas. Maceió, Alagoas, Brasil: Edufal, 2013.

Escohotado, Antonio. Historia de las drogas. Madrid, España: Alianza Editorial, 1998.

Marx, Karl. O capital: crítica da Economia Política. Livro primeiro. Tomo 2. São Paulo, Brasil: Editora Nova Cultural, 2002.

Marx, Karl. A ideologia alemã. São Paulo, Brasil: Expressão Popular, 2005.

\section{PUBLICACIONES PERIÓDICAS}

Almeida, Antonio; Ferreira, Márcia; Barbosa, Maria da Luz; da Silva, Rafael y Santos, Tânia. "O adolescente e as drogas: consequências para a saúde". Escola Anna Nery 11 (4). Río de Janeiro, Brasil, 2007: 605-610.

Cáceres, Delcy; Salazar, Isabel; Varela, María y Tovar, José. "Consumo de drogas en jóvenes universitarios y su relación de riesgo y protección con los factores psicosociales”. Revista Univ. Psycol. 5 (3). Bogotá, Colombia. Octubre-diciembre 2006: 521-534.

Comisión Interamericana para el Control del Abuso de Drogas (CICAD). "Evaluación del Progreso de Control de Drogas 2005-2006". Mecanismo de Evaluación Multilateral. Colombia: Organización de los Estados Americanos (OEA), 2009.

Dávila, Oscar. "Adolescencia y juventud: de las nociones a los abordajes". Última década 12 (21). Valparaíso, Chile. Centro de Estudios Sociales CIDPA, 2005: 83-104.

Jansen, Ney. "Drogas, imperialismo e luta de classes”. Revista Urutágua 12 (1). Brasil. Departamento de Ciências SociaisUniversidade Estadual de Maringá, 2007: 45-56.

Marques, Ana Cecília y Cruz, Marcelo. "O adolescente e o uso de drogas". Revista Brasileira de Psiquiatria 22 (2). São Paulo, Brasil, 2000: 32-36.

Medina, Núbia y Ferriani, Maria das Graças. "Factores protectores de las familias para prevenir el consumo de drogas en un municipio de Colombia”. Revista Latino- 
Americana de Enfermagem 18 (2). São Paulo, Brasil. Universidade de São Paulo, 2010: 504-512.

Murillo-Castro Ligia y Miasso, Adriana. "Visión de jóvenes costarricenses, de zonas rurales, en un programa de rehabilitación, sobre el consumo de drogas". Revista Latino-Americana de Enfermagem 19 (2). São Paulo, Brasil. Universidade de São Paulo, 2011: 96-803.

Netto, José Paulo. "Cinco notas a propósito da questão social". Revista Temporalis 3 (1). Brasília, Brasil. Associação Brasileira de Ensino e pesquisa em Serviço Social, 2001:12-24.

Riofrío, Rosa y Nascimento, Lucila Castanheira. "Consumo de drogas en los jóvenes de la ciudad de Guayaquil, Ecuador". Revista Latino-Americana de Enfermagem 18(1). São Paulo, Brasil. Universidade de São Paulo, 2010: 598-605.

Schenker, Miriam y Minayo, Maria Cecília. "Fatores de risco e de proteção para o uso de drogas na adolescência". Ciência \& Saúde Coletiva 10 (3). Brasil. Associação Brasileira de Pós-Graduação em Saúde Coletiva. Junio-setiembre 2005: 707-717.

Da Silva, Eroy Aparecida; Micheli, Denise De; Camargo, Beatriz Marra Vaz de; Buscatti, Delmara; Alencar, Marlene Asevêdo Passos de y Formigoni, Maria Lucia Oliveira Souza. "Drogas na adolescência: temores e reações dos pais". Psicologia: teoria e prática 8 (1). Brasil. Universidade Presbiteriana Mackenzie, 2006: 41-54.

Velazquez Altamirano, Marina; Arellanez Hernandez, Jorge Luis y Martinez Garcia, Arlette. "Asertividad y consumo de drogas en estudiantes mexicanos". Acta Colombiana de Psicología. 15 (1). Bogotá, Colombia, enero-junio 2012: 131-141.

Fecha de ingreso: 07/07/2014 Fecha de aprobación: 03/11/2014 
